\title{
The efficacy and safety of niraparib for ovarian cancer: a single-center observational study from China
}

Jing $\mathrm{Ni}^{1+}$, Xianzhong Cheng ${ }^{1+}$, Qian Zhao ${ }^{1}$, Zhiqin Dai ${ }^{1}$, Xia Xu², Wenwen Guo ${ }^{3}$, Hongyuan Gu', Rui Zhou', Yan Wang ${ }^{3^{*}}$ and Xiaoxiang Chen ${ }^{1 *}$ (D)

\begin{abstract}
Background: Niraparib, a poly (ADP-ribose) polymerase (PARP) inhibitor, is approved for first/second-line maintenance treatment of ovarian cancer patients with complete or partial response to platinum-based chemotherapy, and multi-line monotherapy in BRCAmt patients or platinum-sensitive recurrence patients with homologous recombination deficiency (HRD). We present real-world experience from a single center of China.

Methods: Patients treated with niraparib in Jiangsu Cancer Hospital between June 2019 to July 2020 were recruited. The initial dose was given according to individualization. Response and adverse events (AEs) were analyzed by Response Evaluation Criteria in Solid Tumors v1.1. and National Cancer Institute Common Terminology Criteria for Adverse Events v5.0, respectively. HRD testing (AmoyDx ${ }^{\oplus}$ ) was detected in most patients. Treatment was given until unequivocal progression or intolerable toxicity.
\end{abstract}

Results: Twenty-two patients all received niraparib at a bolus of $200 \mathrm{mg} / \mathrm{d}$. Fifty percent of patients with high-grade serous ovarian cancer are HRD-positive. Six patients underwent first-line maintenance therapy. Sixteen patients received exploratory therapy. Ultimately image evaluation revealed that two patients achieved partial response (PR) and one patient achieved stable disease (SD), yielding objective response rate (ORR) of $33.3 \%(95 \% \mathrm{Cl}=0.060-0.759)$ and disease control rate $(\mathrm{DCR})$ of $50 \%(95 \% \mathrm{Cl}=0.140-0.861)$ in the exploratory multi-line monotherapy group. The most common AEs were nausea, thrombocytopenia, and anemia. Grade 3-4 thrombocytopenia were managed by dose reduction and interruption. Leg swelling was observed as a new adverse event.

Conclusion: It is feasible that patients receiving a bolus of $200 \mathrm{mg} / \mathrm{d}$ in patients from Chinese population can acquire promising efficacy and tolerance. This is the first real-world data about niraparib in ovarian cancer patients with available HRD status from China.

Keywords: Ovarian cancer, Niraparib, Real world, Efficacy, Safety, HRD

\footnotetext{
*Correspondence: cxxxxcyd@gmail.com; wangyan_9999@sina.com

${ }^{\dagger}$ Jing $\mathrm{Ni}$ and Xianzhong Cheng contributed equally to this work.

'Department of Gynecologic Oncology, Jiangsu Cancer Hospital, Jiangsu

Institute of Cancer Research, The Affiliated Cancer Hospital of Nanjing

Medical University, 42\# Baiziting street, Nanjing, Jiangsu 210009, People's

Republic of China

${ }^{3}$ Department of Pathology, The Second Affiliated Hospital of Nanjing Medical

University, 121\# Jiangjiayuan Road, Nanjing, Jiangsu 210011, People's

Republic of China

Full list of author information is available at the end of the article
}

(c) The Author(s). 2021 Open Access This article is licensed under a Creative Commons Attribution 4.0 International License, which permits use, sharing, adaptation, distribution and reproduction in any medium or format, as long as you give appropriate credit to the original author(s) and the source, provide a link to the Creative Commons licence, and indicate if changes were made. The images or other third party material in this article are included in the article's Creative Commons licence, unless indicated otherwise in a credit line to the material. If material is not included in the article's Creative Commons licence and your intended use is not permitted by statutory regulation or exceeds the permitted use, you will need to obtain permission directly from the copyright holder. To view a copy of this licence, visit http://creativecommons.org/licenses/by/4.0/. The Creative Commons Public Domain Dedication waiver (http://creativecommons.org/publicdomain/zero/1.0/) applies to the data made available in this article, unless otherwise stated in a credit line to the data. 


\section{Background}

Ovarian cancer is the most lethal gynecological malignancy, $70 \%$ of which are diagnosed with advanced stage. Although most ovarian cancer patients are sensitive to standard first-line treatment including cytoreductive surgery and platinum-based chemotherapy, about $80 \%$ patients relapse within 1 to 2 years after initial treatment and gradually progress to platinum-resistance ovarian cancer, accompanied by significantly shortened survival $[1,2]$. How to prolong the platinum free interval (PFI) becomes one of the breakthrough points in ovarian cancer treatment. Recently, poly ADP-ribose Polymerase (PARP) inhibitors have changed the treatment paradigm for ovarian cancer that can significantly improve the PFI, and finally prolonged the overall survival of patients with BRCA mutation [3-6].

PARP is a specific DNA fracture receptor, which is activated after DNA damage. PARP can recognize and bind to the DNA fracture site, mediating DNA singlestrand damage repair in tumor cells [7]. PARP inhibitor can lead to DNA double strand damage inducing by amount of DNA single-strand damage. Normal cells can repair double-strand breaks through homologous recombination repair pathway. In tumor cells with homologous recombination deficiency (HRD), there are several genetic mutations such as BRCA mutation or other mutations in genes of homologous recombination repair (HRR) pathway (e.g., RAD51 and ATM). Due to treatment with PARP inhibitor, tumor cells can't repair DNA single-strand damage and double-strand breaks, forming the synthetic lethal effect [8]. Therefore, BRCAmt or HRD-positive tumor cells are more sensitive to PARP inhibitors in terms of molecular mechanisms.

Niraparib (Zejula ${ }^{\circledR}$ is a highly selective inhibitor of PARP1/2 (nuclear proteins that detect DNA damage and promote its repair) [9]. In 2017, it was firstly approved for second-line maintenance treatment of ovarian cancer patients who were in complete or partial response to platinum-based chemotherapy by Food and Drug Administration (FDA) according to the study of NOVA [3, 10]. Another study observed significant efficacy of niraparib in patients with newly diagnosed advanced ovarian cancer after response to first-line platinum-based chemotherapy [5]. Both of NOVA and PRIMA studies found that patients with HRD could get more benefits from niraparib. Recently the first fully powered, multicenter, phase III clinical study in Chinese population (NORA) showed that median PFS was significantly longer for niraparib as second-line maintenance treatment versus placebo among patients with germline BRCA mutations (not reached vs. 5.5 months; HR: 0.22) and those without germline BRCA mutations (11.1 vs. 3.9 months, HR: 0.40) in 2020 ESMO meeting [11]. QUADRA study demonstrated that niraparib brought great survival benefits among women with heavily pretreated ovarian cancer, especially in patients with HRD-positive platinum-sensitive disease, which included not only patients with BRCA mutation but also population with BRCA wild-type [12].

However, there was no real-world data to illustrate the efficacy and safety of niraparib in Chinese population. We conducted this study to assess the real-world experiences of niraparib in ovarian cancer patients with HRD status from our single center.

\section{Materials and methods \\ Study population}

Patients with ovarian cancer receiving niraparib from June 2019 to July 2020 in Jiangsu Cancer Hospital were included. We collected the baseline characteristics of these patients, including age, Eastern Cooperative Oncology Group performance status (ECOG PS) before the beginning of the treatment, histological type, clinical stage on the basis of International Federation of Gynecology and Obstetrics (FIGO), basal body weight, basal platelet count, previous therapy before and after niraparib treatment and the follow-up. The study was approved by the ethics committee of Jiangsu Cancer Hospital.

\section{Group standard}

Patients who progressed during initial treatment, or completely/partially responded to initial treatment (cytoreductive surgery and platinum-based chemotherapy), but recurred within 6 months are defined as platinum-refractory/platinum-resistant ovarian cancer. Patients relapsed at more than 6 months after initial treatment are considered as platinum-sensitive ovarian cancer. According to the guidelines, patients treated with three or more prior lines of chemotherapy and whose cancer is associated with HRD positive defined by either: 1) a deleterious or suspected deleterious BRCA mutation; or 2) genomic instability and progression more than 6 months after response to the last platinum-based chemotherapy can use niraparib as multi-line therapy.

\section{Dosing regimen}

The initial dose was based on the level of basal body weight and platelet count. Patients with basal body weight $\geq 77 \mathrm{~kg}$ and basal platelet count of $\geq 150,000 / \mu \mathrm{l}$ $(\mu \mathrm{L})$ received $300 \mathrm{mg}$ daily. While patients with basal body weight $<77 \mathrm{~kg}$ and/or basal platelet count $<150$, $000 / \mu \mathrm{L}$ received $200 \mathrm{mg}$ daily. Dose reduction $(300 \mathrm{mg}$ to $200 \mathrm{mg}$ or $100 \mathrm{mg} ; 200 \mathrm{mg}$ to $100 \mathrm{mg}$ ) or interruption for drug-related AEs was allowed. Serum CA125 and imaging examinations were performed on each patient at baseline, followed by monthly examination of CA125 and bimonthly imaging examinations. 


\section{HRD testing}

The paraffin sections from the cytoreductive surgery were obtained after patients' informed consent. DNA was extracted from FFPE biopsy/surgical specimens; 50 to 200 ng DNA undergoes library construction and hybrid capture with AmoyDx ${ }^{\oplus}$ HD panel, which selected coding sequences (CDS) regions for 54 HRR pathway genes and 72,000 single nucleotide polymorphisms (SNPs) for HRD calling. The selected libraries were pooled and sequenced on the Illumina Novaseq6000 to $>500 \times$ unique coverage for 54 HRR genes and $>100 \times$ for SNP loci.

Sequence data was processed using a customized analysis pipeline designed to accurately detect multiple classes of genomic alterations: base substitutions, short insertions/deletions with detection sensitivity at variant allele frequency (VAF) $\geq 5 \%$. Detected mutations were annotated according to American College of Medical Genetics (ACMG) guideline [13] and classified as pathogenic, likely pathogenic, variants of unknown significance, likely benign and benign. HRD score was calculated by the sum of three types of genomic instable events including loss of heterozygosity $(\mathrm{LOH})$, telomeric allelic imbalance (TAI) and large-scale state transition (LST) defined by ref. [14]. HRD-positive was defined by either BRCA1/2 pathogenic or likely pathogenic mutation or HRD score $\geq 42$.

\section{Assessments}

Demographic and baseline data were summarized and analyzed. The efficacy was assessed as complete response $(\mathrm{CR})$, partial response (PR), stable disease (SD) and progressive disease (PD) by RECIST 1.1. Objective response rate (ORR) was defined as the proportion of patients achieving CR or PR. Disease control rate (DCR) was defined as the proportion of patients achieving CR, PR or SD for at least 8 weeks. Treatment-related adverse events (AEs) were graded according to CTCAE 5.0.

\section{Statistical analysis}

The 95\% confidence interval was calculated using the Wilson procedure with a correction for continuity. Data were statistically analyzed using SPSS version 19.0 professional statistical software and all the count data were expressed as a percentage (\%).

\section{Results}

\section{Patients' characteristics}

A total of 22 patients treated with niraparib were enrolled, included 21 patients with ovarian cancer and 1 patient with fallopian tube cancer. The median age was 55.0 years (range 39-77 years). Patient demographics and baseline characteristics were listed in Table 1. FIGO stage II, III and IV, affected 2 (9.1\%), 11 (50.0\%) and 8
(36.4\%) of patients, respectively. Most patients (86.4\%) were high-grade serous cancer. All participants weighed less than $77 \mathrm{~kg}, 10$ of who had basal platelet count less than 150,000 per cubic millimeter. The results of HRD testing were positive in six patients and negative in eight patients.

\section{Group assignment}

On the basis of group standard, the patients were divided into first-line maintenance treatment group and exploratory treatment group (not in the scope of indications), with 6 and 16 patients in each group respectively. For exploratory therapy, there were three subgroups including exploratory second-line maintenance, front-line (less than three chemotherapy regimens) and multi-line (three or more prior chemotherapy regimens) treatment group, with one, six and nine patients in each group, respectively.

\section{Efficacy, CA125 and HRD status}

In first-line maintenance treatment group, all six patients are still on medication, the median follow-up time of whom is 18 weeks (range 8-44 weeks). Among these, two cases were HRD-positive while HRD test was not conducted for the rest four patients. In the exploratory treatment group, one HRD-negative patient diagnosed with highly differentiated papillary mesothelioma has been receiving niraparib as the second-line maintenance treatment. Two cases in exploratory front-line therapy and 9 cases in exploratory multi-line therapy were also tested for HRD. The remaining patients were not underwent HRD tests. Serum CA125 of these patients with maintenance treatment were shown in Fig. 1.

Among the different therapeutic strategies in the exploratory first-line therapy subgroup, three patients who did not undergo surgery and only received chemotherapy for their personal willingness achieved SD after treated with niraparib, of these 2 patients with HRD positive and HRD negative, respectively. All three patients are still assessed as SD. Two patients with first platinum-sensitive recurrence ovarian cancer achieved $\mathrm{SD}$, one of which was treated with niraparib monotherapy and the other one was treated with niraparib and anlotinib. One patient who did not receive chemotherapy after the surgery due to poor renal function achieved PD.

The median prior line was 5 (range 3-8) in the exploratory multi-line therapy subgroup. Ultimately therapeutic evaluation showed that two patients achieved partial response (PR), one patient achieved stable disease (SD) and three patients had progressive disease (PD), yielding the objective response rate (ORR) of $33.3 \%$ $(95 \% \mathrm{CI}=0.060-0.759)$ and the disease control rate (DCR) of $50 \%(95 \% \mathrm{CI}=0.140-0.861)$ in patients with 
Table 1 Baseline characteristics in 22 patients. Values are reported as frequency ( $\mathrm{n}[\%])$ or as mean (range)

\begin{tabular}{|c|c|}
\hline Characteristic & $\begin{array}{l}\text { Number of patients } \\
\text { (percent) }\end{array}$ \\
\hline \multicolumn{2}{|l|}{ Age, yrs } \\
\hline Median age (range) & $55(39-77)$ \\
\hline$\leq 55$ & $12(54.5)$ \\
\hline$>55$ & $10(45.5)$ \\
\hline \multicolumn{2}{|l|}{ Primary tumor location } \\
\hline Ovary & $21(95.5)$ \\
\hline Fallopian tube & $1(4.5)$ \\
\hline \multicolumn{2}{|l|}{ International FIGO stage } \\
\hline$\|$ & $2(9.1)$ \\
\hline$\|$ & $11(50.0)$ \\
\hline IV & $8(36.4)$ \\
\hline Unknown & $1(4.5)$ \\
\hline \multicolumn{2}{|l|}{ Histological type } \\
\hline High-grade serous & $19(86.4)$ \\
\hline Low-grade serous & $1(4.5)$ \\
\hline Other & $1(4.5)$ \\
\hline Unknown & $1(4.5)$ \\
\hline \multicolumn{2}{|l|}{ Family history of cancer } \\
\hline Yes & $9(40.9)$ \\
\hline No & $13(59.1)$ \\
\hline \multicolumn{2}{|l|}{ ECOG } \\
\hline 0 & $8(36.4)$ \\
\hline 1 & $13(59.1)$ \\
\hline 2 & $1(4.5)$ \\
\hline \multicolumn{2}{|l|}{ Baseline body weight } \\
\hline$\geq 77 \mathrm{~kg}$ & $0(0)$ \\
\hline$<77 \mathrm{~kg}$ & $22(100)$ \\
\hline \multicolumn{2}{|l|}{ Platelet count } \\
\hline$\geq 150 \times 10^{9} / \mathrm{L}$ & $12(54.5)$ \\
\hline$<150 \times 10^{9} / \mathrm{L}$ & $10(45.5)$ \\
\hline \multicolumn{2}{|l|}{ HRD status } \\
\hline HRD-positive & $6(27.3)$ \\
\hline BRCA-mutated & $1(4.5)$ \\
\hline $\begin{array}{l}\text { BRCA-wild type or BRCA-unknown and HRD- } \\
\text { positive }\end{array}$ & $5(22.7)$ \\
\hline HRD-negative & $8(36.4)$ \\
\hline HRD unknown & $8(36.4)$ \\
\hline \multicolumn{2}{|l|}{ Prior lines of chemotherapy } \\
\hline$\leq 1$ & $12(54.5)$ \\
\hline$>1$ & $10(45.5)$ \\
\hline
\end{tabular}

Table 1 Baseline characteristics in 22 patients. Values are reported as frequency (n [\%]) or as mean (range) (Continued)

\begin{tabular}{|c|c|}
\hline Characteristic & $\begin{array}{l}\text { Number of patients } \\
\text { (percent) }\end{array}$ \\
\hline \multicolumn{2}{|l|}{ Platinum status } \\
\hline Platinum-sensitive & $5(22.7)$ \\
\hline Platinum-resistant & $5(22.7)$ \\
\hline Unknown & $12(54.5)$ \\
\hline \multicolumn{2}{|l|}{ Categories of therapy } \\
\hline First-line maintenance therapy & $6(27.3)$ \\
\hline Exploratory therapy & $16(72.7)$ \\
\hline $\begin{array}{l}\text { Exploratory second-line maintenance } \\
\text { therapy }\end{array}$ & $1(4.5)$ \\
\hline Exploratory front-line therapy & $6(27.3)$ \\
\hline Exploratory multi-line therapy & $9(40.9)$ \\
\hline \multicolumn{2}{|l|}{ NACT+IDS } \\
\hline Yes & $7(31.8)$ \\
\hline No & $15(68.2)$ \\
\hline \multicolumn{2}{|l|}{ Primary debulking surgery } \\
\hline Yes & $12(54.5)$ \\
\hline No & $10(45.5)$ \\
\hline \multicolumn{2}{|l|}{ Secondary cytoreductive surgery } \\
\hline Yes & $3(13.6)$ \\
\hline No & $19(86.4)$ \\
\hline \multicolumn{2}{|l|}{ Combination with other agents } \\
\hline Yes & $4(18.2)$ \\
\hline No & $18(81.8)$ \\
\hline
\end{tabular}

Abbreviations: FIGO International Federation of Gynecology and Obstetrics, ECOG Eastern Cooperative Oncology Group, HRD homologous recombination deficiency, NACT Neoadjuvant chemotherapy, IDS Interval debulking surgery

exploratory multi-line monotherapy. There were also three patients treated with exploratory multi-line combination therapy, one patient of whom achieved SD using niraparib combined with topotecan and anlotinib but another one failed with this combination therapy. The remaining patient achieved PD by niraparib combined with anlotinib (Table 2). The available HRD status and tumor shrinkage in the exploratory front-line and multi-line treatment subgroups were listed in Fig. 2.

\section{Safety}

The most common AEs were nausea (55.6\%), thrombocytopenia (44.4\%), anemia (33.3\%), and decreased appetite (33.3\%), while serious AEs (SAEs) were thrombocytopenia (16.7\%), anemia (5.6\%), neutropenia (5.6\%), vomiting (5.6\%) and dyspepsia (5.6\%) in patients with niraparib monotherapy. Three patients with thrombocytopenia, neutropenia, or vomiting relieved through dose interruption followed by dose reduction (200 mg to $100 \mathrm{mg}$ ). One patient with dyspepsia could not 


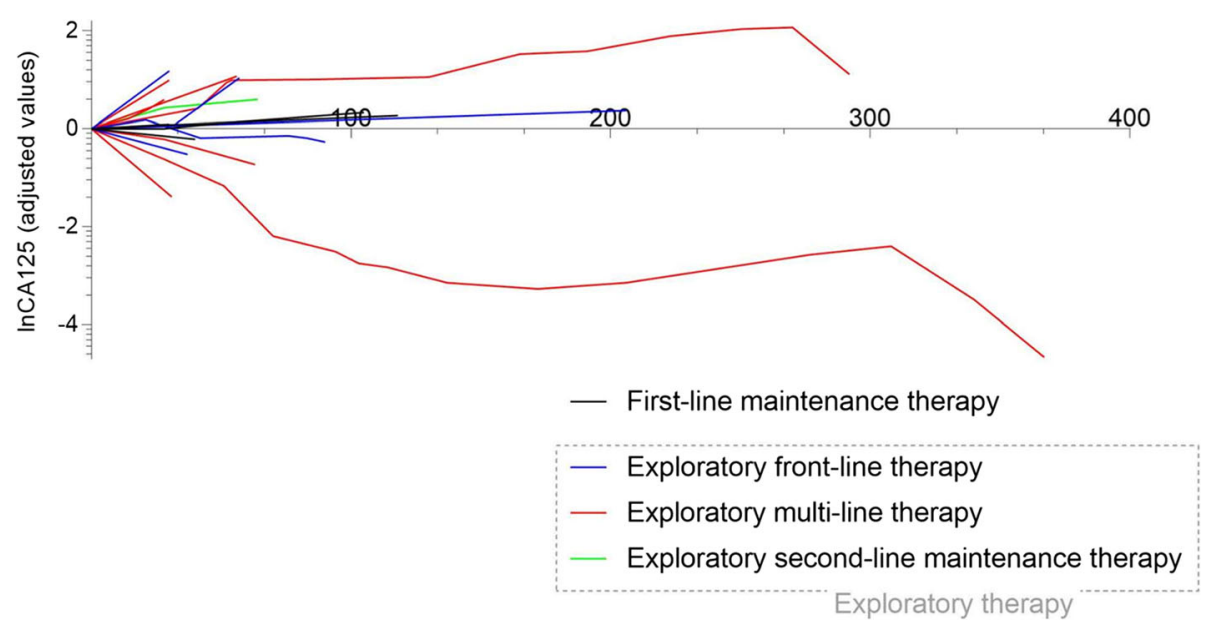

Fig. 1 Serum CA125 values in each group. Note: The CA125 level of the first follow-up was used as the reference value, and all data were converted to natural logarithm. CA125 follow-up data were obtained from 6 patient in the first-line maintenance treatment group and 16 patients in the exploratory therapy group

tolerate drug-related $\mathrm{AE}$ after dose reduction and discontinued the treatment. In patients with niraparib combined therapy, the most common AEs were thrombocytopenia (75.0\%), anemia (75.0\%), fatigue or asthenia (75.0\%), nausea (50.0\%) and neutropenia (50.0\%), while SAEs were thrombocytopenia (25.0\%), anemia $(25.0 \%)$ and neutropenia $(25.0 \%)$. One patient received the combination therapy including niraparib, topotecan and anlotinib suffered grade 4 thrombocytopenia within 1 week. She stopped taking the medicine and were treated with niraparib monotherapy at a dose of $200 \mathrm{mg}$ daily after elevation of platelet count. Another patient treated with niraparib in combination with topotecan and anlotinib also suffered grade3-4 anemia and had a dose reduction and interruption. The remaining two patients treated with niraparib plus anlotinib suffered grade1-2 AEs. SAEs were not observed in two patients with initial dose of $100 \mathrm{mg}$ daily. We observed leg swelling as a new adverse event in one patient. Summary of AEs including niraparib monotherapy and niraparib combined therapy were listed in Table 3.

\section{Discussion}

PARP inhibitor is a major advance in the treatment of ovarian cancer. Patients with BRCA mutation or HRD positive can get more benefit from it. Now there are two kinds of PARP inhibitors including olaparib and niraparib in China. We previously reported the first realword study of olaparib in Chinese population [15]. Here we presented the first real-word experience of niraparib for ovarian cancer patients from China.

A retrospective analysis of ENGOT-OV16/NOVA trial suggested that patients with baseline body weight $<77 \mathrm{~kg}$ or baseline platelets $<150,000 / \mathrm{ml}$ might benefit from a starting dose of $200 \mathrm{mg}$ daily [16]. A subsequent study proved that incidence of common reported AEs of clinical trials were lower among patients initiating niraparib $200 \mathrm{mg} / \mathrm{d}$ in real-world practice versus patients initiating niraparib $300 \mathrm{mg} / \mathrm{d}$ in Caucasian population from ENGOT-OV16/NOVA study [17]. Recently the NORA trial demonstrated that niraparib maintenance therapy administered with an individual starting dose regimen, most subjects received the initial dose of $200 \mathrm{mg} / \mathrm{d}$, significantly improved the outcome in patients with

Table 2 Short-term efficacy of 9 evaluable patients with exploratory multi-line therapy

\begin{tabular}{lll}
\hline Short-term efficacy & Monotherapy, $\mathbf{n}(\%)$ & Combined Treatment, $\mathbf{n}(\%)$ \\
\hline Complete response (CR) & $0(0)$ & $0(0)$ \\
Partial response (PR) & $2(33.3)$ & $0(0)$ \\
Stable disease (SD) & $1(16.7)$ & $1(33.3)$ \\
Progression disease (PD) & $3(50.0)$ & $2(66.7)$ \\
Objective response rate (ORR) & $2 / 6(33.3)$ & $0(0)$ \\
Disease control rate (DCR) & $3 / 6(50.0)$ & $1 / 3(33.3)$
\end{tabular}

The table above showed the short-term efficacy of 9 evaluable patients with exploratory multi-line therapy including 6 patients with niraparib monotherapy and 3 patients with combined treatment. Short-term efficacy was classified by modified Response Evaluation Criteria in Solid Tumors version 1.1 (RECIST 1.1) 


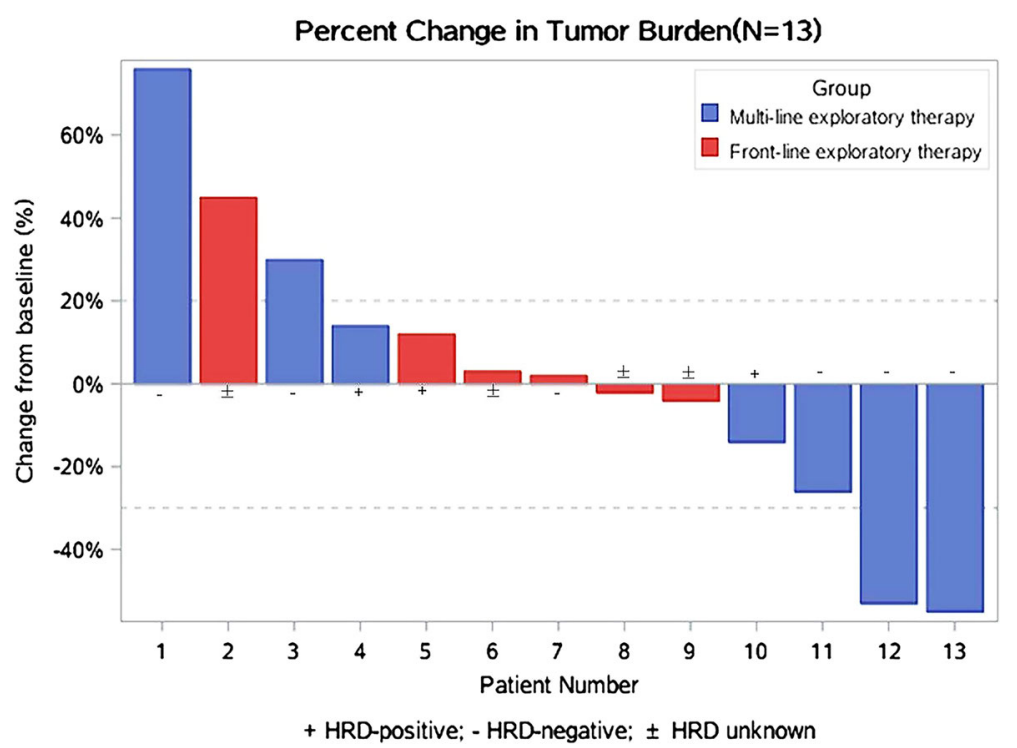

Fig. 2 Waterfall plot of 13 evaluable patients with exploratory therapy. Note: Tumor burden change per investigator review. Maximum reduction from baseline (or smallest increase from baseline for patients with no reductions) in the sum of the longest diameters of target lesions. The change from baseline in tumor measurement as assessed by investigator review is shown for 13 evaluable patients (per protocol set). Front-line exploratory subgroup and multi-line exploratory subgroup were shown with different color in the figure. Among them, there were $3 \mathrm{HRD}$ positive patients, 6 HRD-negative patients and 4 HRD-unknown patients. Ten of them were treated by niraparib monotherapy while 3 of them (patient $1,7,11)$ were treated by combined strategies. The dotted line represents the threshold for partial response $(>30 \%$ reduction from baseline sum of longest diameters) and progressive disease (> 20\% increase from baseline sum of longest diameters). Target lesions were defined according to RECIST 1.1

recurrent ovarian cancer in 2020 ESMO meeting [11]. All patients in our real-world experience receiving 200 $\mathrm{mg} / \mathrm{d}$ according to the basal weight and basal platelet count was consistent with the results of prospective studies in Chinese population.

At the 2020 ASCO meeting, an open-label, nonrandomized study (LIGHT) showed that patients with platinum-sensitive recurrence, high-grade serous/endometrioid epithelial ovarian cancer and one or more prior lines of platinum chemotherapy could benefit from olaparib monotherapy, especially in patients with HRDpositive [18]. Similarly, in our exploratory front-line therapy subgroup, two patients with first platinumsensitive recurrence achieved SD, one of which was with HRD-positive and the other was with HRD-negative. This finding needs to be confirmed by a prospective study of niraparib as front-line monotherapy.

The PAOLA-1 trial suggested that olaparib combined with bevacizumab provided a significant progression-free survival benefit to advanced ovarian cancer patients receiving first-line standard therapy including bevacizumab, which was substantial in patients with HRD positive, including those without BRCA mutation [19]. Niraparib plus bevacizumab significantly improved progression-free survival compared with niraparib alone in platinum-sensitive recurrent ovarian cancer [20]. One HRD-negative patient also achieved SD receiving niraparib plus anlotinib which might be related to the synergistic antitumor effect of PARP inhibitors and antiangiogenic drugs. Further studies are also needed to observe efficacy of the combination strategies in patient with HRD negative, regardless of front-line therapy or multi-line therapy.

A multi-center, open-label, single-arm, phase 2 QUADRA trial observed that 10 (27\%) of 37 platinumresistant patients harbored BRCA mutation, 12 (10\%) of 120 platinum-resistant patients with HRD positive and 5 (3\%) of 169 platinum-resistant patients with HRD negative achieved an overall response according to RECI ST1.1 in the primary analysis of efficacy [12]. Regardless of the patients' BRCA status or HRD status, our results showed the ORR of $33.3 \%(95 \% \mathrm{CI}=0.060-0.759)$ and the DCR of $50 \%(95 \% \mathrm{CI}=0.140-0.861)$ respectively in platinum-resistant ovarian cancer patients with exploratory multi-line monotherapy. We consider the differences of response to niraparib may be due to the small number of patients enrolled in our study and the criteria for enrollment in our real-world data.

Previous studies confirmed that ovarian cancer patients with HRD positive were more likely to benefit from niraparib than those with HRD negative. We didn't observe the obvious relationship between efficacy and HRD status among our available cases, which may be correlated with our small sample size. One study 
Table 3 Summary of adverse events

\begin{tabular}{|c|c|c|c|c|}
\hline \multirow[t]{3}{*}{ Adverse event } & \multicolumn{2}{|c|}{ Niraparib monotherapy $(n=18)$} & \multicolumn{2}{|c|}{ Niraparib combined therapy $(n=4)$} \\
\hline & Any grade & Grade 3 or 4 & Any grade & Grade 3 or 4 \\
\hline & \multicolumn{4}{|c|}{ Number of patients (percent) } \\
\hline Nausea & $10(55.6)$ & $0(0)$ & $2(50.0)$ & $0(0)$ \\
\hline Thrombocytopenia & $8(44.4)$ & $3(16.7)$ & $3(75.0)$ & $1(25.0)$ \\
\hline Anemia & $6(33.3)$ & $1(5.6)$ & $3(75.0)$ & $1(25.0)$ \\
\hline Decreased appetite & $6(33.3)$ & $0(0)$ & $1(25.0)$ & $0(0)$ \\
\hline Fatigue or asthenia & $5(27.8)$ & $0(0)$ & $3(75.0)$ & $0(0)$ \\
\hline Constipation & $5(27.8)$ & $0(0)$ & $1(25.0)$ & $0(0)$ \\
\hline Insomnia & $5(27.8)$ & $0(0)$ & $0(0)$ & $0(0)$ \\
\hline Neutropenia & $3(16.7)$ & $1(5.6)$ & $2(50.0)$ & $1(25.0)$ \\
\hline Vomiting & $3(16.7)$ & $1(5.6)$ & $0(0)$ & $0(0)$ \\
\hline Dyspepsia & $3(16.7)$ & $1(5.6)$ & $0(0)$ & $0(0)$ \\
\hline Headache & $2(11.1)$ & $0(0)$ & $1(25.0)$ & $0(0)$ \\
\hline Abdominal distention & $2(11.1)$ & $0(0)$ & $0(0)$ & $0(0)$ \\
\hline Dizziness & $1(5.6)$ & $0(0)$ & $1(25.0)$ & $0(0)$ \\
\hline Dysgeusia & $1(5.6)$ & $0(0)$ & $0(0)$ & $0(0)$ \\
\hline Back pain & $1(5.6)$ & $0(0)$ & $0(0)$ & $0(0)$ \\
\hline Diarrhea & $1(5.6)$ & $0(0)$ & $0(0)$ & $0(0)$ \\
\hline Maculopapular rash & $1(5.6)$ & $0(0)$ & $0(0)$ & $0(0)$ \\
\hline Stomatitis & $1(5.6)$ & $0(0)$ & $0(0)$ & $0(0)$ \\
\hline Dry mouth & $1(5.6)$ & $0(0)$ & $0(0)$ & $0(0)$ \\
\hline Abdominal pain & $0(0)$ & $0(0)$ & $1(25.0)$ & $0(0)$ \\
\hline \multicolumn{5}{|l|}{ Newly observed } \\
\hline Leg swelling & $1(5.6)$ & $0(0)$ & $0(0)$ & $0(0)$ \\
\hline Led to discontinuation of intervention & $1(5.6)$ & - & $0(0)$ & - \\
\hline Led to dose reduction & $3(16.7)$ & - & $1(25.0)$ & - \\
\hline Led to dose interruption & $2(11.1)$ & - & $2(50.0)$ & - \\
\hline
\end{tabular}

Note: Adverse events were graded according to National Cancer Institute Common Terminology Criteria for Adverse Events (NCI CTCAE), version 5.0

reported that approximately $50 \%$ of patients with highgrade serous ovarian cancer are HRD-positive [21]. Among all enrolled cases in our study, 21 participants of whom were BRCAwt or BRCA unknown, and 14 cases were tested with HRD panel. Of the 12 patients with high-grade serous ovarian cancer, 6 patients were with HRD- positive and 6 patients were HRD-negative. The results of our report were consistent with the study of large sample.

The most common AEs were nausea, thrombocytopenia, anemia and fatigue. SAEs were thrombocytopenia, anemia, neutropenia and dyspepsia for both niraparib monotherapy and combined therapy that was higher in latter. The incidence of AEs and SAEs in our observation were similar to other studies. A meta-analysis of current clinical trials also showed the same characteristics of AEs [22]. All SAEs occurred within 1 month after receiving therapy with niraparib, most of which occurred within 1 week. The most common AEs caused dose reduction and interruption of treatment were serious thrombocytopenia and vomiting. We also observed that one patient suffered grade 1 leg swelling in the second week after treatment with niraparib $200 \mathrm{mg}$ daily, the mechanism of which need to be explored in future.

Severe myelosuppression including thrombocytopenia and neutropenia, and vomiting were alleviated by dose reduction and interruption. Only one patient with dyspepsia could not tolerate after dose reduction to 100 $\mathrm{mg} / \mathrm{d}$ and discontinued the treatment. With respect to PARP inhibitors combined with chemotherapy, the GOLD study did not meet its primary objective of showing a significant improvement in overall survival with olaparib in combination with a chemotherapeutic agent and in the overall or ATM-negative population of Asian patients with advanced gastric cancer due to the intolerable AEs [23]. In our study, one patient was given the 
combination therapy including niraparib, topotecan and anlotinib. She suffered grade 4 thrombocytopenia within 1 week. Similar to the GOLD study, we also observed the intolerable AEs in patients using PARP inhibitors combined with chemotherapy.

\section{Conclusion}

This is the first real word data about niraparib in ovarian cancer patients with HRD status from China. Our findings demonstrated that Chinese population with niraparib $200 \mathrm{mg}$ orally once daily is feasible. Leg swelling was observed as a new adverse event in our study. HRD tests in our small samples confirmed that $50 \%$ of patients with high-grade serous ovarian cancer were HRDpositive. However, our data are limited representative due to limited number of cases. Further clinical trials are needed to verify the exploratory therapy in our study.

\section{Abbreviations}

PARP: Poly (ADP-ribose) polymerase; HRD: Homologous recombination deficiency; HRR: Homologous recombination repair; PFI: Platinum-freeinterval; RECIST: Response Evaluation Criteria in Solid Tumors

\section{Acknowledgments}

Not applicable.

\begin{abstract}
Authors' contributions
Jing Ni participated in the design of present study and drafted the manuscript. Xianzhong Cheng carried out the cases recruit of present study. Qian Zhao and Hongyuan Gu participated in the cases recruit of present study. Wenwen Guo and Rui Zhou carried out statistical analysis. Zhiqin Dai and Xia Xu participated in the statistical analysis and drafted the manuscript. Yan Wang participated in decision of molecular pathology. Xiaoxiang Chen designed of the study, performed the statistical analysis and revised the manuscript. All authors read and approved the final manuscript.
\end{abstract}

\section{Funding}

This study was supported by grants from the National Natural Science Foundation of China (No. 81472441, 81501205), Institute level project of Jiangsu Cancer Hospital (No. ZM201804), Beijing Kanghua Foundation for the Development of Traditional Chinese and Western Medicine -Le Fund $(\mathrm{KH}$ 2020-LJJ-021) and Jiangsu Province Maternal and Child Health Research Project (F202004).

\section{Availability of data and materials}

We would not share the data and material used in this manuscript, because we need them for further research.

\section{Declarations}

\section{Ethics approval and consent to participate}

This study was approved by the institutional review board of Jiangsu Cancer Hospital, Nanjing Medical University, China. The informed consent requirement was waived. The committee's reference number was Jiangsu Cancer Hospital's Ethical Committee 2020-068.

\section{Consent for publication}

Not applicable.

\section{Competing interests}

The authors declare that they have no competing interests.

\section{Author details}

'Department of Gynecologic Oncology, Jiangsu Cancer Hospital, Jiangsu Institute of Cancer Research, The Affiliated Cancer Hospital of Nanjing Medical University, 42\# Baiziting street, Nanjing, Jiangsu 210009, People's
Republic of China. '2Department of Chemotherapy, Nanjing Medical University Affiliated Cancer Hospital, Jiangsu Cancer Hospital, Jiangsu Institute of Cancer Research, 42\# Baiziting street, Nanjing, Jiangsu 210009, People's Republic of China. ${ }^{3}$ Department of Pathology, The Second Affiliated Hospital of Nanjing Medical University, 121\# Jiangjiayuan Road, Nanjing, Jiangsu 210011, People's Republic of China.

Received: 31 October 2020 Accepted: 5 April 2021

Published online: 17 May 2021

\section{References}

1. Coleridge SL, et al. Chemotherapy versus surgery for initial treatment in advanced ovarian epithelial cancer. Cochrane Database Syst Rev. 2019;10: CD005343.

2. Guan LY, Lu Y. New developments in molecular targeted therapy of ovarian cancer. Discov Med. 2018;26(144):219-29.

3. Mirza MR, Monk BJ, Herrstedt J, Oza AM, Mahner S, Redondo A, et al. Niraparib maintenance therapy in platinum-sensitive, recurrent ovarian cancer. N Engl J Med. 2016;375(22):2154-64. https://doi.org/10.1056/ NEJMoa1611310.

4. Ledermann J, Harter P, Gourley C, Friedlander M, Vergote I, Rustin G, et al. Olaparib maintenance therapy in platinum-sensitive relapsed ovarian cancer. N Engl J Med. 2012;366(15):1382-92. https://doi.org/10.1056/ NEJMoa1105535.

5. González-Martín A, Pothuri B, Vergote I, DePont Christensen R, Graybill W, Mirza MR, et al. Niraparib in patients with newly diagnosed advanced ovarian cancer. N Engl J Med. 2019;381(25):2391-402. https://doi.org/10.1 056/NEJMoa1910962.

6. Poveda A, et al. Final overall survival (OS) results from SOLO2/ENGOT-ov21: a phase III trial assessing maintenance olaparib in patients (pts) with platinum-sensitive, relapsed ovarian cancer and a BRCA mutation. Proc Am Soc Clin Oncol. 2020.

7. Murai J, Huang SYN, Das BB, Renaud A, Zhang Y, Doroshow JH, et al. Trapping of PARP1 and PARP2 by clinical PARP inhibitors. Cancer Res. 2012; 72(21):5588-99. https://doi.org/10.1158/0008-5472.CAN-12-2753.

8. Schiewer MJ, et al. PARP-1 regulates DNA repair factor availability. EMBO Mol Med. 2018;10(12):e8816.

9. Heo YA, Duggan ST. Niraparib: a review in ovarian cancer. Target Oncol. 2018;13(4):533-9. https://doi.org/10.1007/s11523-018-0582-1.

10. Ison G, Howie LJ, Amiri-Kordestani L, Zhang L, Tang S, Sridhara R, et al. FDA approval summary: niraparib for the maintenance treatment of patients with recurrent ovarian cancer in response to platinum-based chemotherapy. Clin Cancer Res. 2018;24(17):4066-71. https://doi.org/10.1158/1078-0432. CCR-18-0042.

11. Wu XH, et al. Individualized starting dose of niraparib in patients with platinum-sensitive recurrent ovarian cancer (NORA) : a randomized, doubleblind, placebo-controlled, phase III trial. Proc Euro Soc Med Oncol. 2020.

12. Moore KN, Secord AA, Geller MA, Miller DS, Cloven N, Fleming GF, et al. Niraparib monotherapy for late-line treatment of ovarian cancer (QUADRA): a multicentre, open-label, single-arm, phase 2 trial. Lancet Oncol. 2019;20(5): 636-48. https://doi.org/10.1016/S1470-2045(19)30029-4.

13. Richards S, et al. Standards and guidelines for the interpretation of sequence variants: a joint consensus recommendation of the American College of Medical Genetics and Genomics and the Association for Molecular Pathology. Genet Med. 2015;17(5):405-24. https://doi.org/10.1038/ gim.2015.30.

14. Timms KM, Abkevich V, Hughes E, Neff C, Reid J, Morris B, et al. Association of BRCA1/2 defects with genomic scores predictive of DNA damage repair deficiency among breast cancer subtypes. Breast Cancer Res. 2014;16(6):475. https://doi.org/10.1186/s13058-014-0475-X.

15. Ni J, Cheng X, Zhou R, Xu X, Guo W, Chen X. Olaparib in the therapy of advanced ovarian cancer: first real world experiences in safety and efficacy from China. J Ovarian Res. 2019;12(1):117. https://doi.org/10.1186/s13048-01 9-0594-1.

16. Berek JS, Matulonis UA, Peen U, Ghatage P, Mahner S, Redondo A, et al. Safety and dose modification for patients receiving niraparib. Ann Oncol. 2018;29(8):1784-92. https://doi.org/10.1093/annonc/mdy181.

17. Gallagher JR, Heap KJ, Carroll S, Travers K, Harrow B, Westin SN. Real-world adverse events with niraparib $200 \mathrm{mg} /$ day maintenance therapy in ovarian cancer: a retrospective study. Future Oncol. 2019;15(36):4197-206. https:// doi.org/10.2217/fon-2019-0471. 
18. Cadoo K, et al. Olaparib treatment in patients (pts) with platinum-sensitive relapsed (PSR) ovarian cancer (OC) by BRCA mutation (BRCAm) and homologous recombination deficiency (HRD) status: phase II LIGHT study. Proc Am Soc Clin Oncol. 2020.

19. Ray-Coquard I, Pautier P, Pignata S, Pérol D, González-Martín A, Berger R, et al. Olaparib plus bevacizumab as first-line maintenance in ovarian cancer. N Engl J Med. 2019;381(25):2416-28. https://doi.org/10.1056/NEJMoa19113 61.

20. Mirza MR, Åvall Lundqvist E, Birrer MJ, dePont Christensen R, Nyvang GB Malander $S$, et al. Niraparib plus bevacizumab versus niraparib alone for platinum-sensitive recurrent ovarian cancer (NSGO-AVANOVA2/ENGOTov24): a randomised, phase 2, superiority trial. Lancet Oncol. 2019;20(10): 1409-19. https://doi.org/10.1016/S1470-2045(19)30515-7.

21. lijima M, et al. Genome-wide analysis of gynecologic cancer: the cancer genome atlas in ovarian and endometrial cancer. Oncol Lett. 2017;13(3): 1063-70.

22. Jiang $\mathrm{YF}$, et al. Evaluation of the efficacy and safety of PARP inhibitors in advanced-stage epithelial ovarian cancer. Front Oncol. 2020;10:954.

23. Bang YJ, Xu RH, Chin K, Lee KW, Park SH, Rha SY, et al. Olaparib in combination with paclitaxel in patients with advanced gastric cancer who have progressed following first-line therapy (GOLD): a double-blind, randomised, placebo-controlled, phase 3 trial. Lancet Oncol. 2017;18(12) 1637-51. https://doi.org/10.1016/S1470-2045(17)30682-4.

\section{Publisher's Note}

Springer Nature remains neutral with regard to jurisdictional claims in published maps and institutional affiliations.

- fast, convenient online submission

- thorough peer review by experienced researchers in your field

- rapid publication on acceptance

- support for research data, including large and complex data types

- gold Open Access which fosters wider collaboration and increased citations

- maximum visibility for your research: over $100 \mathrm{M}$ website views per year

At $\mathrm{BMC}$, research is always in progress.

Learn more biomedcentral.com/submissions 